\title{
DETERMINAÇÃO DE COEFICIENTES CONVECTIVOS DE TRANSFERÊNCIA DE CALOR POR MEIO DAS TÉCNICAS DE FLUIDODINÂMICA COMPUTACIONAL
}

\author{
J. P. S. SILVA, D. O. SILVA e L. G. M. VIEIRA \\ Universidade Federal de Uberlândia, Faculdade de Engenharia Química \\ E-mail: luizgustavo@feq.ufu.br
}

\begin{abstract}
RESUMO - Os coeficientes convectivos de transferência de calor são essenciais para projeto e controle de processos térmicos que envolvam o mecanismo de convecção. A avaliação experimental dos coeficientes de película não é algo trivial porque são parâmetros que dependem simultaneamente da geometria do sistema, das características fluidodinâmicas do escoamento e das propriedades físico-químicas do fluido. Assim, uma interessante alternativa para estimar os coeficientes convectivos de transferência de calor seria aplicar técnicas de CFD, nas quais as Equações de Transporte são resolvidas numérica e simultaneamente. Logo, este trabalho teve como principal objetivo aplicar as técnicas de CFD para avaliar os coeficientes de película em uma superfície esférica, compará-los com valores advindos da literatura e correlacioná-los em função dos Números de Re e Pr. De acordo com os principais resultados, a metodologia em CFD foi viável e apresentou satisfatórios resultados para previsão dos coeficientes convectivos de transferência de calor.
\end{abstract}

\section{INTRODUÇÃO}

Todas as formas de energia são importantes na atuação profissional do engenheiro. Porém, em se tratando de Fenômenos de Transporte, a atenção é direcionada à transferência de calor (energia de origem térmica) que pode se dar por meio de três mecanismos: condução, convecção e radiação. Dentre tais mecanismos, verifica-se que o transporte de calor por convecção requer especial tratamento devido ao fato de estar intrinsecamente acoplado ao transporte de quantidade de movimento. A convecção de calor somente é possível devido a gradientes térmicos verificados no interior de um fluido ou entre este e determinada interface (sólido/líquido ou líquido/líquido), responsáveis pelo escoamento (natural ou forçado) do fluido ao longo do sistema. Em tese, todos os gradientes térmicos ou taxas de calor em determinado fluido poderiam ser calculados sob a óptica dos Fenômenos de Transporte, mediante a solução conjunta da Equação da Energia Térmica com a Equação do Movimento e a Equação da Continuidade (subsidiadas por uma Equação de Estado e Correlações para descrição de outras propriedades físicas em função da temperatura e pressão). Na prática a solução mencionada anteriormente não é nada trivial devido à complexidade matemática e computacional requerida pelo fenômeno.

Assim, uma alternativa muito empregada para a descrição dos efeitos da convecção está centrada na Lei de Resfriamento de Newton (Equação 1). A Lei de Resfriamento de Newton está baseada em uma equação algébrica relativamente simples que tenta correlacionar a taxa do calor transportado com grandezas físicas de percepção imediata (área disponível à transferência e gradiente térmico), mediante a compatibilização por uma constante de proporcionalidade. 


$$
\mathrm{q}=\mathrm{hA}\left(\mathrm{T}-\mathrm{T}_{\infty}\right)
$$

Na Lei de Resfriamento de Newton, a constante de proporcionalidade (h) é denominada de coeficiente convectivo de transferência de calor (CCTC) ou simplesmente de coeficiente de película (Bird, 2004). Pragmaticamente, o conhecimento do CCTC é essencial para o respectivo projeto de equipamentos (evaporadores, trocadores de calor, reatores, destiladores, caldeiras etc.), cujo desconhecimento inviabilizaria qualquer tomada de decisão (projeto ou controle). O CCTC não é uma grandeza unicamente ligada às propriedades da matéria, mas também dependente das características do escoamento (Burmeister, 1983). Assim, antes dos projetos de equipamentos térmicos propriamente ditos, é conditio sine qua non que os coeficientes de películas sejam necessariamente conhecidos ou passíveis de determinação (Kaviany, 1994). Tradicionalmente, a determinação dos coeficientes de película ocorre de maneira experimental (Churchill, 1973), exigindo instrumentação e investimentos financeiros relativamente onerosos. Atualmente, devido aos avanços da fluidodinâmica computacional (Patankar, 1980) é possível avaliar propriedades de escoamentos a partir das Equações de Transporte (Maliska, 1995) em softwares que fazem uso de técnicas numéricas específicas, comumente englobados sob a denominação de CFD (Computational Fluid Dynamics). Isto posto, este trabalho teve como principal objetivo utilizar-se das técnicas de CFD para verificar a viabilidade de estimativa numérica dos coeficientes convectivos médios de transferência de calor em superfícies esféricas, a fim de que, no futuro, se procedente tal metodologia, ela possa ser generalizada e também aplicada a outras geometrias, inclusive complexas.

\section{METODOLOGIA}

A fim de que as técnicas de Fluidodinâmica Computacional (CFD) pudessem ser adequadamente aplicadas na determinação numérica dos coeficientes convectivos de transferência de calor (CCTC), este trabalho adotou um planejamento de "experimentos numéricos", no qual três distintos Números de Reynolds (Re) foram combinados com outros três diferentes Números de Prandtl (Pr), a fim de cobrir uma significativa faixa operacional. O Número de Reynolds foi calculado com base no diâmetro da esfera e as propriedades físicas do fluido necessárias aos adimensionais (viscosidade, densidade, calor específico e condutividade térmica) foram estimadas na temperatura de aproximação do fluido $\left(\mathrm{T}_{\infty}\right)$. Em se tratando do número de Prandtl, a variação deste adimensional somente foi possível mediante a utilização de diferentes fluidos. Além disso, a variação deste adimensional não foi igualmente espaçada (manipulada) pelo fato dela depender intrinsecamente da natureza físico-química do fluido. Isto posto, na Tabela 1 são apresentados as nove simulações realizadas neste trabalho.

Tabela 1 - Combinações entre Re e Pr utilizadas para levantamento dos CCTC em CFD

\begin{tabular}{|c|c|c|c|c|c|c|c|c|}
\hline $\mathbf{N}$ & Fluido (Pr) & Re & $\mathbf{N}$ & Fluido (Pr) & Re & $\mathbf{N}$ & Fluido (Pr) & Re \\
\hline $\mathbf{0 1}$ & $\operatorname{ar}(0,74)$ & 100 & $\mathbf{0 4}$ & querosene $(28)$ & 100 & $\mathbf{0 7}$ & Etileno glicol $(150)$ & 100 \\
\hline $\mathbf{0 2}$ & $\operatorname{ar}(0,74)$ & 5050 & $\mathbf{0 5}$ & querosene $(28)$ & 5050 & $\mathbf{0 8}$ & Etileno glicol $(150)$ & 5050 \\
\hline $\mathbf{0 3}$ & $\operatorname{ar}(0,74)$ & 10000 & $\mathbf{0 6}$ & querosene $(28)$ & 100000 & $\mathbf{0 9}$ & Etileno glicol $(150)$ & 100000 \\
\hline
\end{tabular}

Nas condições da Tabela 1, os fatores Pr e Re foram codificados (Equações 2 e 3 ) visando à possível regressão múltipla, a fim de avaliar possíveis efeitos existentes entre eles (lineares, quadráticos e de interação). Neste caso, o ar, o querosene e o etileno glicol foram codificados para $-1,0000 ;-0,6347$ e $+1,0000$, respectivamente. Já os regimes de escoamento de 100, 5050 e 10000 
(calculados com base no diâmetro da esfera) foram codificados para -1, 0 e +1 , respectivamente. As equações para cálculo dos adimensionais também são apresentadas (Equações 4 e 5).

$$
\begin{aligned}
& X_{1}=\frac{\operatorname{Pr}-75,37}{74,63} \\
& \operatorname{Pr}=\frac{\mu_{\infty} \mathrm{cp}_{\infty}}{\mathrm{k}_{\infty}}
\end{aligned}
$$

$$
\begin{aligned}
& X_{2}=\frac{\operatorname{Re}-5050}{4950} \\
& \operatorname{Re}=\frac{\rho_{\infty} \mathrm{vd}}{\mathrm{u}_{\infty}}
\end{aligned}
$$

Uma malha computacional foi gerada no software GAMBIT ${ }^{\circledR}$ 2.3.16 para um "túnel de vento" cilíndrico de comprimento $\mathrm{L}(0,20 \mathrm{~m})$ e diâmetro $\mathrm{D}(0,20 \mathrm{~m})$, no qual uma esfera maciça de diâmetro $\mathrm{d}(0,02 \mathrm{~m})$ foi inserida exatamente na metade do eixo do cilindro. A malha computacional utilizada nesse trabalho foi tridimensional, refinada próximo à superfície esférica e constituída por aproximadamente $2.10^{5}$ células tetraédricas. Este número de células foi obtido após um teste de independência de malha no qual esta quantidade não proporcionava mais qualquer variação nas propriedades do escoamento (Petri Jr. et al., 2012). Por sua vez, as simulações em CFD foram realizadas no software comercial FLUENT $^{\circledR}$ 14.0, cujas condições numérico-operacionais utilizadas estão mencionadas na Tabela 2.

Tabela 2 - Condições numérico-operacionais empregadas para as simulações do escoamento do fluido ao longo do "túnel de vento"

\begin{tabular}{l|l} 
Fluido & ar \\
\hline Temperatura de Aproximação do Fluido $\left(\mathrm{T}_{\infty}\right)$ & $298 \mathrm{~K}$ \\
\hline Temperatura do Sólido $\left(\mathrm{T}_{\mathrm{S}}\right)$ & $398 \mathrm{~K}$ \\
\hline Modelo de Turbulência & LES (Large Edge Simulation) \\
\hline Passo de Integração Temporal & $0,1 \mathrm{~s}$ \\
\hline Critério de Convergência & $1 \times 10^{-4}$ \\
\hline Algoritmo de Acoplamento Pressão-Velocidade & SIMPLE \\
\hline Esquema de Interpolação & QUICK \\
\hline Método de Determinação de h & Surface Integrals \\
\hline Pressão de Descarga do Túnel de Vento & 95245 Pa (Uberlândia-MG) \\
\hline Direção do escoamento & vertical e contrário à gravidade
\end{tabular}

De posse dos valores gerados pelo FLUENT ${ }^{\circledR} 14.0$ (função Surface Integrals), foi possível aplicar sobre eles as técnicas de Superfície de Resposta (Box et al., 1978), no intuito de se obter os parâmetros de uma função matemática (correlação) que descrevesse o comportamento do CCTC médio (h) para quaisquer valores de $\operatorname{Re}\left(\mathrm{X}_{1}\right)$ e $\operatorname{Pr}\left(\mathrm{X}_{2}\right)$ que estivessem dentro do domínio inicialmente proposto (Myers, 1976), cuja forma genérica pode ser representada pela Equação (6). A Equação (6) tem como parâmetros a serem estimados: um coeficiente independente ou médio $(\beta)$, coeficientes de interação linear $\left(b_{1}\right.$ e $\left.b_{2}\right)$, coeficientes de interação quadrática $\left(B_{11}, B_{22}\right)$ e um coeficiente de interação $\left(\mathrm{B}_{12}=\mathrm{B}_{21}\right)$.

$$
h=\beta+\left(\begin{array}{ll}
b_{1} & b_{2}
\end{array}\right)\left(\begin{array}{l}
X_{1} \\
X_{2}
\end{array}\right)+\left(\begin{array}{ll}
X_{1} & X_{2}
\end{array}\right)\left(\begin{array}{cc}
B_{11} & \frac{B_{12}}{2} \\
\frac{B_{21}}{2} & B_{22}
\end{array}\right)\left(\begin{array}{l}
X_{1} \\
X_{2}
\end{array}\right)
$$


Por fim, vale mencionar que, antes do levantamento da superfície de resposta (Equação 6), os valores de CCTC obtidos pela presente metodologia (CFD) foram comparados com aqueles previstos por meio da Correlação de Whitaker (1972). Tal correlação tem natureza empírica e representa um clássico da literatura para estimativa de CCTC em geometrias esféricas submetidas ao escoamento com gases. Se uma vez verificada a plausibilidade desta metodologia em CFD para o gás, bem provável também seria o êxito para a respectiva aplicação nos escoamentos com líquidos (querosene e etileno glicol).

\section{RESULTADOS}

A fim de se verificar a metodologia aqui proposta em CFD, algumas simulações computacionais foram realizadas (independentemente daqueles já previstos na Tabela 1), cujos valores de coeficientes convectivos de transferência de calor médios (CCTC) estimados pela função Integrals Surface do Fluent foram comparados com a Correlação de Whitaker (aplicáveis a escoamentos gasosos e geometria esférica). Tais resultados encontram-se dispostos na Figura 1.

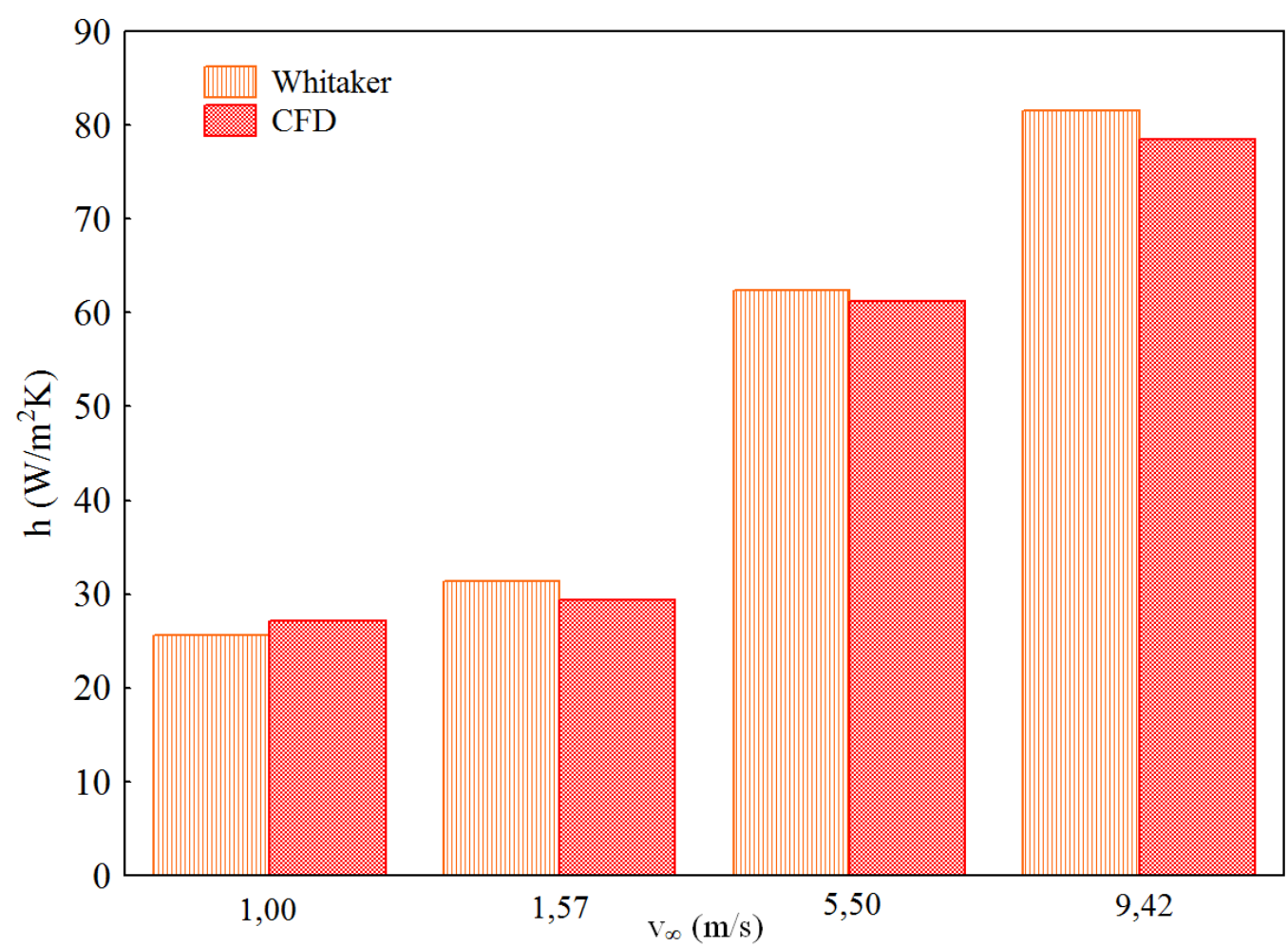

Figura 1 - Valores de CCTC (h) estimados pelas técnicas de CFD e pela Correlação de Whitaker.

De acordo com a Figura 1, foi possível observar que as técnicas de CFD foram viáveis porque conseguiram prever satisfatoriamente os valores dos coeficientes convectivos médios de transferência de calor (h) para esferas (diferença média de 2,33\% em relação à correlação de Whitaker). Apesar de Whitaker (1972) não apresentar os intervalos de confiança dos valores de $h$ preditos por sua correlação que, em tese, poderiam até contemplar os valores estimados pelas técnicas de CFD desse trabalho, acredita-se que as técnicas de CFD são estatisticamente equivalentes, permitindo, portanto, sua aplicação a outros resfriamentos feitos por diferentes fluidos (querosene e etileno glicol). 
A título de exemplo, são apresentados na Figura 2 o perfil de velocidade e a distribuição térmica do fluido ao redor da esfera, respectivamente, para a linha $\mathrm{N}=3$ da Matriz de Planejamento (Tabela 1). De acordo com a primeira parte da Figura 2, foi possível verificar que a esfera, em função de sua localização espacial, esteve sujeita a uma distribuição uniforme de velocidade a sua montante, indicando que a razão entre o diâmetro da esfera e do túnel de vento foi apropriada para os objetivos desse trabalho $(\mathrm{d} / \mathrm{D}=0,10)$. Já nas proximidades da esfera, foi possível observar que houve uma forte interação entre o gás e a superfície sólida. Em um primeiro momento, houve a formação de uma camada limite à montante da estrutura sólida, enquanto que à jusante começou a haver um descolamento sob a forma de esteiras de Von Kàrman, indicando, portanto, a necessidade de realmente ter sido empregado um modelo de turbulência como o LES (Large Edge Simulation) para a descrição físico-matemática desse escoamento.

De maneira análoga ao perfil de velocidade, foi possível observar também a coerência para a distribuição de temperatura no fluido ao redor da esfera. De acordo a segunda parte da Figura 2, observou-se que, à montante da esfera, o fluido foi aquecido subitamente, haja vista que, nesta região, a transferência de calor é sempre a mais eficaz devido à existência dos maiores gradientes térmicos. Conforme o escoamento avançou sobre esfera, as temperaturas do fluido tenderam a diminuir por causa dos efeitos da turbulência que provocam mistura entre parcelas de fluido quente com as de fluido frio (descolamento da camada limite), de modo que, a uma distância razoável à jusante da esfera, a temperatura foi novamente uniformizada para valores próximos à temperatura de aproximação do fluido $\left(\mathrm{T}_{\infty}\right)$.

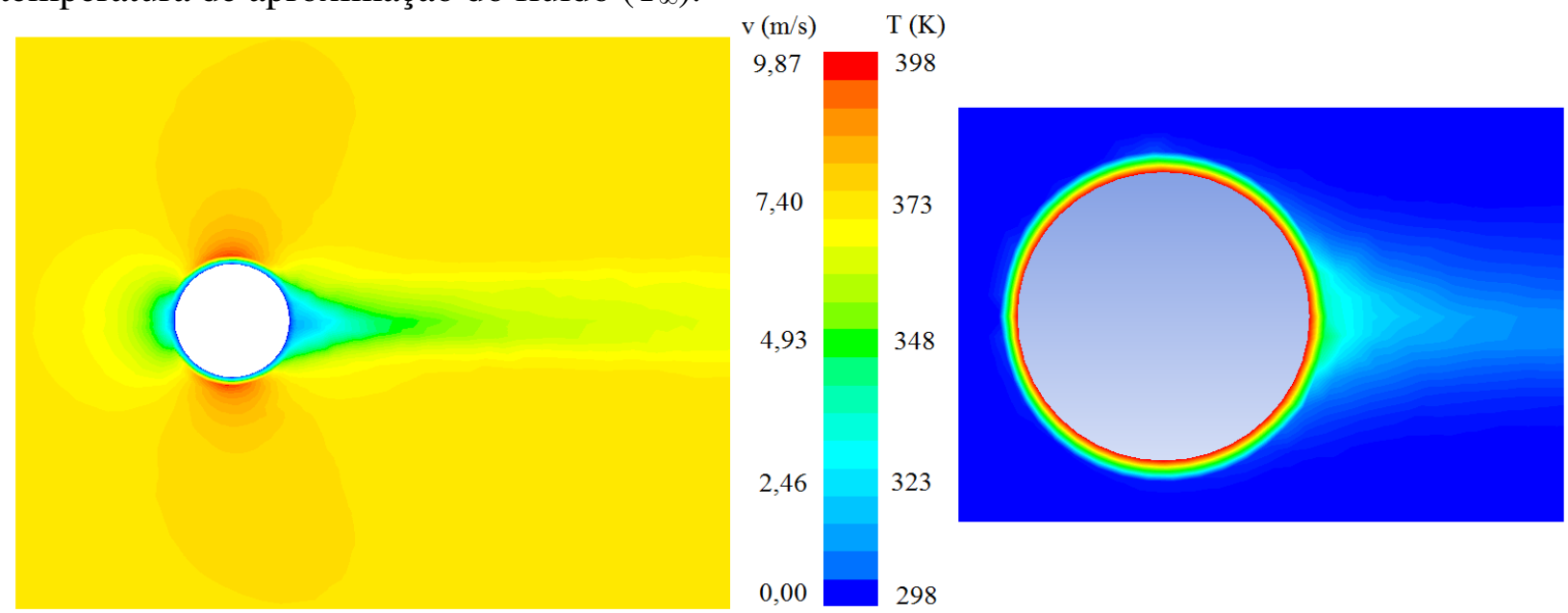

Figura 2 - Perfil de velocidade e distribuição térmica do fluido ao redor da esfera para $\operatorname{Re}=10000$ e $\operatorname{Pr}=0,74$

Aplicando a metodologia descrita neste trabalho para a Matriz de Planejamento da Tabela 1, foi possível, após as simulações em CFD, estimar para cada um dos experimentos numéricos, o coeficiente convectivo médio de transferência de calor médio (h) através da função Integrals Surface do Fluent. Os valores obtidos pela simulação encontram-se dispostos na Figura 3, na qual verificou-se que tanto o regime de escoamento quanto o tipo de fluido de resfriamento exerceram influência direta no coeficiente convectivo de calor. Todavia, para se quantificar os efeitos de cada fator sobre o CCTC, necessário foi o levantamento da Superfície de Resposta para esta grandeza. 


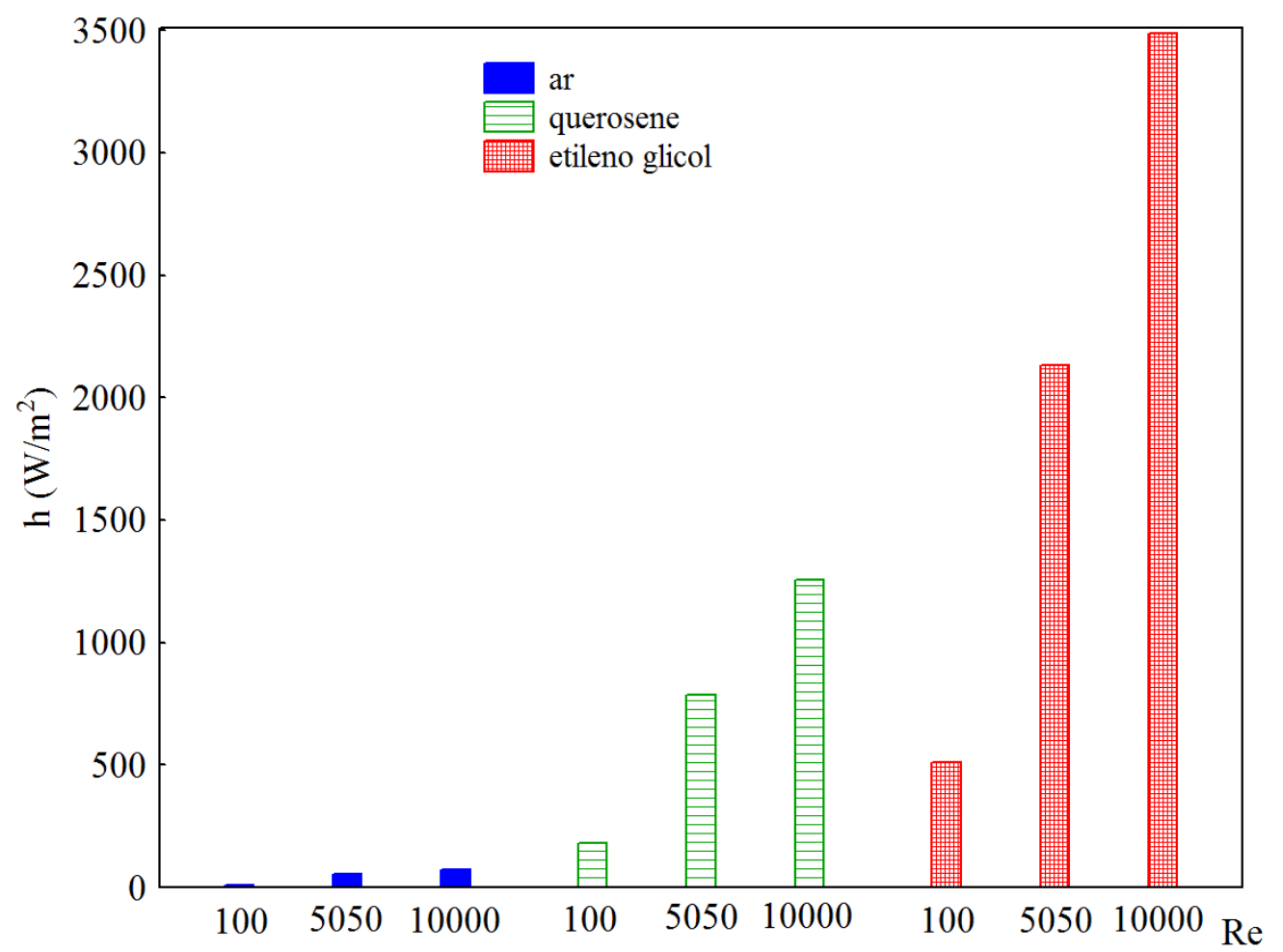

Figura 3 - Coeficientes Convectivos de Transferência de Calor médios (h) obtidos via CFD a partir da combinação de Pr e Re da Matriz de Planejamento (Tabela 1).

Para o levantamento da Superfície de Resposta, a partir dos valores de CCTC estimados pelas técnicas de CFD da Figura 3, Técnicas de Regressão Múltipla (Box et al., 1978) foram aplicadas para o ajuste dos parâmetros da Superfície de Resposta que relaciona o coeficiente convectivo médio de transferência de calor (h) com o número de Reynolds e o número de Prandtl, cujos valores podem ser vistos na Equação 7 (versão decodificada da Equação 6). Vale mencionar que somente foram considerados os parâmetros com nível de significância igual ou menor que 5\%, sendo que após a exclusão dos parâmetros não significativos, a Equação ainda manteve uma confiabilidade ou variância de 99,27\%.

$$
h=1600+\left(\begin{array}{ll}
1000 & 831
\end{array}\right)\left(\begin{array}{l}
\frac{\operatorname{Pr}-75,37}{74,63} \\
\frac{\operatorname{Re}-5050}{4950}
\end{array}\right)+\left(\begin{array}{ll}
\frac{\operatorname{Pr}-75,37}{74,63} & \frac{\operatorname{Re}-5050}{4950}
\end{array}\right)\left(\begin{array}{cc}
-555 & 341 \\
341 & 0
\end{array}\right)\left(\begin{array}{c}
\frac{\operatorname{Pr}-75,37}{74,63} \\
\frac{\operatorname{Re}-5050}{4950}
\end{array}\right)
$$

$$
0,74 \leq \operatorname{Pr} \leq 150 \text { e } 100 \leq \operatorname{Re} \leq 10000
$$

De acordo com a equação de Superfície de Resposta (Equação 7), foi possível verificar que tanto o Número de Prandtl (Pr) quanto o Número de Reynolds (Re) foram efeitos que afetaram diretamente os valores de CCTC. Apesar da importância de ambos, restou esclarecido que o tipo de fluido utilizado para o escoamento foi o efeito preponderante na transferência de calor, haja vista a comparação da ordem de grandeza entre os efeitos lineares de $\operatorname{Pr}$ e $\operatorname{Re}(1000$ e 831 , respectivamente). Desta forma, fica evidente o motivo pelo qual os CCTC do etileno glicol (maiores Prandtl) foram, em média, 45 vezes maior quando comparado ao resfriamento com ar. $\mathrm{O}$ 
mesmo raciocínio se aplica ao querosene que apresentou CCTC por volta de 16 vezes maiores que aqueles verificados para o ar. No que tange aos efeitos quadráticos, apenas o Número de Prandtl foi significativo para o CCTC. Além disso, houve também um efeito de interação entre os fatores estudados, representado pela diagonal secundária da matriz quadrada contida na Equação 7.

De posse de todas as discussões apresentadas anteriormente, pôde-se verificar que as técnicas de Fluidodinâmica Computacional (CFD) foram capazes de prever com confiabilidade satisfatória os valores dos coeficientes convectivos médios de transferência de calor. Tal fato representou um avanço na área de transferência de calor por convecção porque esta metodologia pode ser generalizada para outras geometrias, inclusive complexas, cuja determinação experimental de h é ainda onerosa, imprecisa ou inviável. Além disso, a superfície de resposta obtida neste trabalho (Equação 5) mostra-se interessante porque contempla em uma mesma estrutura uma ampla faixa de operação para Re e Pr, bem como uma forma generalizada aplicável tanto para gases como líquidos.

\section{CONCLUSÕES}

De acordo com os principais resultados numéricos obtidos neste trabalho, foi possível constatar que as técnicas de CFD foram uma interessante ferramenta para também se estimar os coeficientes convectivos de transferência de calor para geometrias esféricas. A partir das simulações fluidodinâmicas, os valores de coeficiente de película numéricos foram calculados e comparados com aqueles oriundos da clássica Correlação Empírica de Whitaker (1972), cujo confronto mostrou um desvio relativo médio de 2,33\% entre os dois procedimentos.

Tanto o Número de Reynolds quanto o Número de Prandtl afetaram diretamente a transferência de calor. Porém, em se tratando de transferência de calor em geometrias esféricas, foi possível concluir que o efeito do tipo de fluido (intrinsecamente relacionado ao Número de Prandtl) foi mais impactante do que o próprio regime de escoamento da fase fluida sobre a superfície sólida (Reynolds). Neste aspecto, foi observado que, para um mesmo regime de escoamento, o resfriamento com etileno glicol (líquido) proporcionou um coeficiente de película cerca de 45 vezes maior do que quando se utilizava ar.

A partir da metodologia numérica proposta neste trabalho, foi possível propor uma correlação mais generalizada do que a de Whitaker (aplicável somente a gases) com uma confiabilidade de 99,27\% para a previsão dos coeficientes de película a partir do conhecimento do Número de Reynolds e do Número de Prandtl.

Por fim, pôde-se concluir que os estudos fluidodinâmicos realizados neste trabalho indicaram que as técnicas de CFD podem ser perfeitamente generalizadas para outras geometrias (inclusive complexas), permitindo aos engenheiros que levantem correlações "numéricas" conforme a geometria de interesse e os fluidos empregados.

\section{NOMENCLATURA}

$\mathrm{A}$ - área disponível à troca térmica na convecção $\left(\mathrm{m}^{2}\right)$

CCTC - abreviação da iniciais de "Coeficiente Convectivo de Transferência de Calor" $\mathrm{cp}_{\infty}$ - calor específico do fluido estimado em $\mathrm{T}_{\infty}(\mathrm{J} / \mathrm{kg})$

$\mathrm{d}$ - diâmetro da esfera maciça $(\mathrm{m})$

D - diâmetro do "túnel de vento" (m)

$\mathrm{h}$ - coeficiente convectivo de transferência de calor $\left(\mathrm{W} / \mathrm{m}^{2 \mathrm{o}} \mathrm{C}\right)$ 
$\mathrm{k}_{\infty}$ - condutividade térmica do fluido estimada em $\mathrm{T}_{\infty}(\mathrm{W} / \mathrm{m} . \mathrm{K})$

$\mathrm{L}$ - comprimento do túnel de vento $(\mathrm{m})$

$\mathrm{N}$ - sequência das simulações numéricas em CFD

Pr - número de Prandtl

q - taxa de calor transportada na convecção (W)

Re - número de Reynolds

$\mathrm{v}$ - velocidade média de aproximação do fluido $(\mathrm{m} / \mathrm{s})$

$\mathrm{T}$ - temperatura do fluido $(\mathrm{K})$

$\mathrm{T}_{\infty}$ - temperatura de aproximação do fluido $(\mathrm{K})$

$\mathrm{X}_{1}$ - codificação do Número de Prandtl

$\mathrm{X}_{2}$ - codificação do Número de Reynolds

$\beta$ - termo independente da Superfície de Resposta $\left(\mathrm{W} / \mathrm{m}^{2} \mathrm{~K}\right)$

$\mu_{\infty}$ - viscosidade dinâmica do fluido estimada em $\mathrm{T}_{\infty}(\mathrm{kg} / \mathrm{m} . \mathrm{s})$

$\rho_{\infty}$ - densidade do fluido estimada em $\mathrm{T}_{\infty}\left(\mathrm{kg} / \mathrm{m}^{3}\right)$

\section{AGRADECIMENTOS}

Os autores deste trabalho agradecem à FAPEMIG (PCE-00082/2014) e ao Laboratório de Separação e Energias Renováveis (LASER) da FEQUI/UFU.

\section{REFERÊNCIAS}

BIRD, R. B.; STEWART, W. E.; LIGHTFOOT, E. N. Fenômenos de Transporte, 2a Ed. 838p. Wiley, New York, 2004

BOX, M. J.; HUNTER, W. G.; HUNTER, J. S. (1978), Statistics for Experiments: An Introdution to Design, Data Analysis and Model Building, $1^{\text {st }}$ Ed. 735p. John Wiley and Sons, New York, 1978.

BURMEISTER, L. C. Convective Heat Transfer, $1^{\text {st }}$ Ed. 650p. Wiley-Interscience, New York, 1983.

CHURCHILL, S. W.; OZOE, H. J. Heat Transfer, 95-78, 1973.

MALISKA, C. R. Transferência de Calor e Mecânica dos Fluidos Computacional: Fundamentos e Coordenadas Generalizadas, $1^{\text {st }}$ Ed. 250 p. LTC, Rio de Janeiro, 1995.

MYERS, R. H. Response Surface Methodology, $1^{\text {st }}$ Ed. 512p. Edwards Brother (Distributors), Virginia, 1976.

PATANKAR, S. V. Numerical Heat Transfer and Fluid Flow, $1^{\text {st }}$ Ed. 248p. Hemisphere Publishing, New York, 1980.

PETRI-JR. I.; COSTA, L. M. A.; VIEIRA, L. G. M. Aplicação das Técnicas de Fluidodinâmica Computacional para Determinação de Coeficientes Convectivos de Transferência de Calor em Geometrias Esféricas. In: Anais do XV Encontro de Modelagem Computacional \& III Encontro de Ciência e Tecnologia de Materiais (EMC \& ECTM), Uberlândia-MG, 2012.

WHITAKER, S. Forced convection heat transfer correlation for flow in pipes, past flat plates, single cylinders, single spheres and flow in packed beds and tube bundles, J. AICHE, 18, 361-371, 1972. 